\title{
Relação entre o genótipo e o temperamento de novilhos em pastejo e seu efeito na qualidade da carne ${ }^{1}$
}

\author{
Isabella Dias Barbosa Silveira², Vivian Fischer ${ }^{3}$, Germano Jorge Dorneles Soares ${ }^{3}$
}

\author{
1 Parte da tese apresentada à UFPel. \\ 2 Pós-Graduação em Zootecnia - UFPel. \\ ${ }^{3}$ Departamento de Zootecnia - UFPel.
}

RESUMO - Avaliaram-se a interação grupo genético $\times$ temperamento e seu efeito sobre a carne de bovinos de corte. Foram utilizados 40 novilhos machos castrados de dois grupos genéticos (Aberdeen Angus - AA e 50\% Aberdeen Angus e 50\% Nelore - NA), com dois anos de idade e peso inicial de 336,4 $\pm 29,39 \mathrm{~kg}$, distribuídos em delineamento completamente casualizado. O temperamento foi avaliado por meio da atribuição de escores comportamentais (EC) e mensuração do tempo de saída (TS). Os novilhos NA apresentaram os maiores EC. Os TS dos animais AA foram maiores que os dos NA nas avaliações 1 e 4 (2,1 $\mathrm{x}$ 1,3 e 4,7 x 2,3 segundos, respectivamente). O ganho médio de peso foi negativamente correlacionado ao ECm e positivamente ao TSm. No grupo genético NA, os animais mais reativos apresentaram menor ganho de peso e aqueles com EC mais altos, menores valores de pH1 $(6,47 ; 6,28$; e 6,09 para EC 1,2 e 3 , respectivamente). Os pH1 mais baixos foram verificados em animais com TS $(6,10 ; 6,43$; e 6,43 para TS 1,2 e 3 , respectivamente). Os animais NA apresentaram pH1 menor que os AA, com 6,17 e 6,57, respectivamente. A carne de novilhos NA com maiores TS foi superior em 23\% para a cor a* $(\mathrm{P}=0,0394)$ e em 9\% para a cor $b^{*}(\mathrm{P}=0,0808)$. A cor $L^{*}$ se correlacionou negativamente com EC3 e pH24 (r=-0,54 e -0,39, respectivamente) e a cor $\mathrm{b}^{*}$, com $\mathrm{pH} 3$ e $\mathrm{pH} 7$ ( $\mathrm{r}=-0,39$ e $-0,45$, respectivamente). Novilhos azebuados e mais agitados apresentam ganho de peso inferior e menores alterações quanto ao $\mathrm{pH}$ inicial e à cor da carne.

Palavras-chave: bovinos, cor, ganho de peso, $\mathrm{pH}$, reatividade

\section{Relation between genotype and temperament of grazing steers on meat quality}

\begin{abstract}
The effect of two genetic groups, Aberdeen angus (AA) and 50\% Nellore x 50\% Aberdeen angus (NA), on temperament and meat quality of 40 steers averaging 2 years of age and $336.4 \pm 29.39 \mathrm{~kg}$ of body weight was evaluated in this trial. Animals were assigned to treatments according to a completely randomized design. Temperament was evaluated through measurements of composite behavior score (BS) and flight time (FT). NA steers had higher BS than AA steers in this trial. Conversely, AA steers had greater FT values than NA steers on evaluations 1 and 4: 2.1 vs. 1.3 e 4.7 vs. 2.3 seconds, respectively. Mean daily weight gain was negatively related with overall BS $(r=-0.47, P=0.0075, n=30)$ while the opposite was observed with overall FT $(\mathrm{r}=0.39, \mathrm{P}=0.0336, \mathrm{n}=30)$. Within the NA group, more reactive animals gained less weight. Steers with higher BS showed lower pH1 values: 6.47, 6.28 and 6.09, respectively, for BS of 1, 2 and 3. Steers with smaller FT showed lower pH1: 6.1, 6.43, and 6.43, respectively, for TF of 1, 2 and 3. Meat of NA steers showed pH1 lower than that of AA steers: 6.17 vs. 6.57. It was observed $23 \%$ more color $\mathrm{a}^{*}(\mathrm{P}=0.0394)$ and $9 \%$ more color $\mathrm{b}^{*}(\mathrm{P}=0.0808)$ on meat from NA steers with larger FT values. Color $\mathrm{L}^{*}$ was negatively related with $\mathrm{BS} 3$ and $\mathrm{pH} 24\left(\mathrm{r}=-0.54\right.$ and -0.39 , respectively) whereas color $\mathrm{b}^{*}$ was negatively related with $\mathrm{pH} 3$ and $\mathrm{pH} 7(\mathrm{r}=-0.39$ and $\mathrm{r}=-0.45$, respectively). Crossbred and more nervous steers had lower weight gain and poorer meat quality.
\end{abstract}

Key Words: bovine, color, $\mathrm{pH}$, reactivity, weight gain

\section{Introdução}

O conhecimento do efeito do genótipo e do temperamento de bovinos de corte na qualidade final do produto pode constituir-se em recurso de utilização prática para os criadores. A produção de carne bovina é uma das principais atividades do agronegócio no estado do Rio Grande do Sul, que possui uma população estimada de 12,99 milhões de bovinos, com taxa de abate projetada em 25,5\% (Anuário da Pecuária Brasileira - Anualpec, 2003).

No Rio Grande do Sul, os rebanhos são compostos basicamente por bovinos pertencentes aos grupos genéticos Bos taurus taurus, denominados Europeus, e Bos taurus taurus x Bos taurus indicus, denominados Cruzas. As criações são, na maioria, extensivas, caracterizadas pelo pequeno contato entre seres humanos e animais. No entanto, 
as operações de rotina nas fazendas, como pesagens, vacinações e transporte, são potencialmente estressantes para os animais e seres humanos e possivelmente refletem na segurança da mão-de-obra, no bem-estar dos animais e na qualidade da carne (Paranhos da Costa, 2002). A identificação dos animais mais ou menos reativos pode permitir a adequação de práticas de manejo, o treinamento de mão-de-obra e, ainda, servir como critério de descarte de animais.

Os bovinos de corte reagem, em diferentes situações, de acordo com o seu temperamento (uma organização hormonal, nervosa e física), que pode ser definido como a percepção e reação dos animais a estímulos que originam medo (Boissy $\&$ Bouissou, 1995) ou ao manejo do homem (Aguilar et al., 2004). Embora seu conceito seja antigo em psicologia, o reconhecimento do seu valor econômico é relativamente recente (Paranhos da Costa et al., 2002; Busby, 2004).

Os fatores genéticos influenciam a intensidade, aumentando ou diminuindo as reações de medo dos animais (Boissy \& Bouissou, 1995). Rogan \& LeDoux (1996) sugerem que o medo é produto de um sistema neural que evoluiu para detectar o perigo e que provoca no animal uma resposta que o proteja.

Em situações de desconforto ou ameaça por algum estímulo (agente estressor), tanto de ambiente (frio, calor, espaço, fome, sede) como de disputas (relação social) ou de manejo (o processo de industrialização da carne compreende desde a saída do animal do seu local de criação até o momento do abate - transporte, manejo pré-abate e abate), o animal pode desencadear uma resposta emocional que se manifesta por mudanças comportamentais, provocando alterações fisiológicas, denominadas genericamente estresse (Grandin, 2000). A resposta ao estresse, nesta situação, é catabólica, tendendo a mobilizar reservas energéticas do organismo para o funcionamento do cérebro e dos músculos.

O processo de conversão do músculo em carne é complexo e envolve uma série de alterações no metabolismo celular e na estrutura protéica, caracterizando-se pelo esgotamento das reservas de trifosfato de adenosina (ATP), pela diminuição do $\mathrm{pH}$ ou acidificação, pela queda da temperatura da musculatura, pelo aumento da concentração de íons cálcio no citosol, pela rigor mortis, entre outros (Lawrie, 1998). O estresse pode determinar alterações no metabolismo muscular antes ou durante o abate, modificando a qualidade final da carne, e resultar em defeitos conhecidos como PSE (pale, soft, exudative) e DFD (dark, firm, dry), ou ainda carne escura (dark cutting).

É provável que o medo seja a causa de grande parte dos efeitos negativos do manejo estressante no rendimento e na saúde dos animais. Petherick et al. (2002) verificaram maior suscetibilidade aos agentes estressores pré-abate de bovinos com temperamento excitável que com temperamento mais calmo.

De acordo com Burrow \& Dillon (1997), animais que saem calmamente dos bretes de contenção ganham mais peso que aqueles que saem mais rapidamente, provavelmente pela maior ingestão de alimentos e da menor suscetibilidade a doenças (Kilgour, 1998). Borba et al. (1997) verificaram, em novilhos sob regime de semiconfinamento, correlações negativas entre distância de fuga e ganho de peso e entre distância de fuga e ganho de peso aos 550 dias de idade. Do mesmo modo, Brown et al. (2004) verificaram correlação negativa entre a velocidade de saída e o ganho de peso médio e entre o peso final e o consumo de matéria seca.

Neste trabalho, avaliaram-se a relação grupo genético versus temperamento e sua influência na qualidade da carne de bovinos de corte.

\section{Material e Métodos}

Foram utilizados 40 bovinos machos castrados, pertencentes a dois grupos genéticos, Bos taurus taurus (Aberdeen Angus, AA, $\mathrm{n}=20$ ) e Bos taurus taurus $x$ Bos taurus indicus $(50 \%$ Nelore $+50 \%$ Aberdeen Angus, NA, $n=20)$, com dois anos de idade e peso inicial de 336,4 $\pm 29,39 \mathrm{~kg}$.

Os animais foram mantidos em campo nativo, suplementado na proporção de $0,5 \%$ do peso vivo, com concentrado comercial $(\mathrm{PB}=15 \%$, cálcio $=1,3 \%, \mathrm{EE}=2,2 \%$, fósforo $=2,75 \%$ ) composto por calcário calcítico, farelo de arroz integral, farelo de soja, farelo de trigo, fosfato bicálcico, milho moído, uréia pecuária, cloreto de sódio e sal mineral).

A cada 28 dias, os animais foram pesados avaliados quanto ao temperamento, por meio da atribuição do escore composto (EC), adaptado de Piovesan (1998), e do tempo de saída (TS), adaptado de Burrow et al. (1988).

A avaliação pelo escore composto (EC) foi realizada durante a pesagem individual dos animais, em seis oportunidades, efetuando-se a última pesagem antes do embarque para o frigorífico. Foram registradas a intensidade de movimentação na balança, a audibilidade da respiração, a presença/ausência de mugidos e a presença/ausência de golpes, atribuindo-se escores para cada um desses comportamentos, conforme a descrição a seguir:

Movimentação na balança (MB):

$1=$ pouco deslocamento dentro da balança, parado na maior parte do tempo, encostado na parte da frente ou de trás, movimentos de cauda ocasionais e relaxados;

2 = animal mais ativo, permanência na mesma posição por poucos segundos, movimentos de cauda ocasionais e vigorosos; 
3 = deslocamento freqüente dentro da balança, movimentos vigorosos e abruptos, movimentos de cauda freqüentes e vigorosos;

4 = deslocamento freqüente dentro da balança, movimentos vigorosos e abruptos, tentativa de se virar; e

5 = deslocamento contínuo, saltos, força a grade de saída com a cabeça, movimentos de cauda contínuos e vigorosos.

Respiração(RESP):

0 = respiração não audível;

$1=$ respiração audível e de forma ritmada (semelhante à respiração habitual);

2 = respiração muito profunda, audível, porém em ritmo diferente à da respiração habitual, com maior tempo de expiração que inspiração; e

3 = bufando e/ou roncando.

Mugidos (MUG):

0 = ausência de mugidos; e

$1=$ ocorrência de um ou mais mugidos.

Golpes (coices e/ou cabeçadas) (GOP):

0 = ausência de golpes; e

$1=$ ocorrências de um ou mais golpes.

Foram utilizadas cinco classes de reatividade, dispostas em ordem crescente, para definir a escala de escore composto (EC) que integrou as avaliações supracitadas. Em uma escala nominal de 1 a 5, os animais com maiores valores de escore foram classificados como reativos.

$1=$ Calmo: escore de movimentação $(\mathrm{MB})=1$; escore de respiração (RESP) 0 ou 1 ; escore de mugidos (MUG) 0 ou 1 ; e escore de golpes (GOP) 0;

2 = Ativo: escore de movimentação 1 (se RESP $\geq 2$ ) ou 2 (se RESP < 2); escore de respiração 0, 1 ou 2 (se MUG ou $\mathrm{GOP}=1$ ) ou 3 ( se MUG ou GOP =0); escores de mugidos 0 ou 1; e escore de golpes 0 ou 1 ;

3 = Inquieto: escore de movimentação 2 (se MUG ou $\mathrm{GOP} \neq 0$ ) ou 3 ( se MUG e GOP=0); escore de respiração 0, 1,2 ( se MUG ou GOP = 1) ou 3 ( se MUG ou GOP=0); escore de mugidos 0 ou 1; e escore de golpes 0 ou 1 ;

4 = Reativo ou muito perturbado: escore de movimentação 3 ( e MUG ou GOP $\neq 0$ ) ou 4 (se MUG ou GOP=0); escore de respiração $0,1,2$ (se MUG ou GOP = 1) ou 3 (se MUG ou $\mathrm{GOP}=0$ ); escore de mugidos $=0$ ou 1 ; e escore de golpes 0 ou 1 ;

5 = Muito reativo ou intratável/perigoso: escore de movimentação 4 (se MUG ou GOP $\neq 0$ ) ou 5 (independentemente dos resultados nos demais escores).

O teste de tempo de saída (TS) foi realizado após cada pesagem dos animais, totalizando seis avaliações considerando-se a rapidez com que os animais saíram após a abertura da porta da balança. Avaliou-se o tempo gasto (em segundos) para que os animais percorressem uma distância de 2,0 m, imediatamente após sair da balança em direção a um espaço aberto. Para o registro desse intervalo, foi utilizado um sistema de células fotoelétricas que acionavam e interrompiam o funcionamento de um cronômetro. Animais com menor tempo foram classificados como mais reativos.

Ao final do período experimental, em razão de uma estiagem ocorrida durante o experimento, somente 30 animais (11 AA e 19 NA) atingiram o peso de $450 \mathrm{~kg}$. Os animais foram embarcados na fazenda em caminhões e transportados até o frigorífico Mercosul, localizado no município de Bagé-RS, a $400 \mathrm{~km}$ da fazenda. O trajeto foi realizado em aproximadamente 6 horas, com início às $11 \mathrm{~h} 40 \mathrm{e}$ término às $18 \mathrm{~h}$, na planta frigorífica.

Os animais foram observados quanto ao temperamento durante o embarque (entrada no caminhão, lotação), o percurso (avaliação do transporte, quedas, paradas para averiguação das condições dos animais) e o descarregamento, (saída do caminhão, quedas, velocidade). Após o descarregamento, os animais foram dirigidos aos currais de espera, para o descanso regulamentar (mínimo de 12 horas) e a dieta hídrica. O manejo dos animais transcorreu de acordo com a rotina da planta frigorífica (pesagem após descarregamento, descanso regulamentar, condução com utilização de bastão elétrico, banho de aspersão, atordoamento e sangria).

Após o abate e durante a sangria, foram coletadas amostras de sangue para determinação do cortisol plasmático. Amostras de $10 \mathrm{~mL}$ de sangue foram colocadas em copos plásticos, que foram mantidos inclinados. Após a separação do soro, as amostras foram congeladas e, posteriormente, encaminhadas para análise em laboratório por radioimunoensaio.

A determinação do $\mathrm{pH}$ foi realizada em amostras de $5 \mathrm{~g}$ do músculo esterno mandibularis, coletadas a 1, 3, 7, 12 e 24 horas post mortem. As amostras foram imediatamente mergulhadas em solução tampão de iodoacetato de sódio ( $5 \mathrm{mM} / \mathrm{KCl})$, com a finalidade de inibir a glicólise post mortem (Bendall, 1973) e, a seguir, foram trituradas e homogeneizadas por 30 segundos em homogenizador Ultraturrax (mod. TE 102, Tecnal, Brasil), estabilizando-se a temperatura do homogenato a $20^{\circ} \mathrm{C}$. A leitura do $\mathrm{pH}$ foi realizada em peagâmetro Analion Mod. PM 602.

Para avaliação da cor, foram retiradas amostras de 100 g do músculo Longissimus dorsi, entre a 12a e 13 costelas, 24 horas post mortem, que foram imediatamente congeladas para posterior leitura em colorímetro Minolta (Minolta Chroma Meter CR-300, Minolta Corp., Ramsey, NJ). Foram estimados os valores de $\mathrm{L}^{*}, \mathrm{a}^{*}, \mathrm{~b}^{*}$, do sistema CIELab, em que L* é o croma associado à luminosidade ( $\mathrm{L}^{*}=0$ preto, 100 branco); $\mathrm{a}^{*}$, o croma que varia do verde 
(-) ao vermelho (+); e b*, o que varia do azul (-) ao amarelo $(+)$ (Houben et al., 2000). Para a leitura no colorímetro, as amostras foram mantidas em repouso com a superfície exposta ao ambiente, por 30 minutos, para a oxigenação da mioglobina (Abularach et al., 1998). As medidas foram realizadas em três regiões diferentes na superfície de interesse, tomando-se a um valor médio.

Foi adotado o delineamento experimental completamente casualizado, em que cada animal compôs uma unidade experimental. Primeiramente, a variável EC foi submetida à análise não-paramétrica de associação linear pelo teste Cochran-Mantel-Haenzel para se verificar a associação global e em cada avaliação entre grupo genético e reatividade. A variável TS foi submetida à análise da variância, considerando o efeito de grupo genético, pelo procedimento GLM, SAS versão 6.12 (1989). Posteriormente, as variáveis $\mathrm{pH}$ (medidas em cada horário), cor, ganho de peso e cortisol foram submetidas à análise de variância, considerando-se os efeitos de grupo genético e EC ou TS e suas respectivas interações. A separação de médias foi realizada pelo Lsmeans, teste DMS Fisher, com nível mínimo de 0,05 de significância para rejeição da hipótese de nulidade. A análise de correlação foi feita utilizando-se o procedimento CORR (Pearson), do programa SAS versão 6.12 (1989), entre o ganho de peso e as variáveis TS e EC medidos em cada avaliação, assim como com os seus valores médios gerais, denominados ganho de peso médio, escore composto médio (Ecm) e tempo de saída médio (TSm).

Para melhor visualização dos resultados em relação ao escore comportamental (EC), os animais foram reagrupados segundo três classes distintas: $1=$ animal mais manso; $2=$ animal intermediário; e 3 = animal nervoso. Para a variável TS os animais foram reagrupados em três classes, conforme a seguir: 1 = animal percorreu em menos tempo a distância de 2 metros (nervoso); 2 = valor intermediário; e 3 = animal que percorreu em maior tempo a distância de 2 metros (calmo).

\section{Resultados e Discussão}

Foram encontradas diferenças significativas entre os grupos genéticos quanto ao escore composto (EC), de modo que os animais AA apresentaram os menores valores em todas as pesagens (Tabela 1). Houve associação linear entre a presença de genética zebu e maiores valores de EC em cada uma das avaliações, considerando-se, de forma global, todas as seis avaliações.

Os resultados indicam que os bovinos NA possuem temperamento mais agitado que os AA e que essas diferenças se mantiveram durante as primeiras cinco avaliações e na avaliação global do experimento.
Os resultados são similares aos obtidos por Grandin (1993), que avaliou, em quatro oportunidades, o temperamento de bovinos cruzados confinados, e verificou que o escore dos animais, segundo o temperamento, não se alterou entre as avaliações. De acordo com Burrow \& Dillon (1997), o manejo de zebuínos e suas cruzas, em condições extensivas, é mais difícil que o dos bovinos europeus, ocorrendo diferenças também entre as raças.

Embora o manejo rotineiro da fazenda tenha sido freqüente e classificado como positivo, ou seja, durante as rotinas de manejo, não houve a presença de cachorros, utilização de guizos ou gritos, a maior reatividade ao manejo verificada nos bovinos NA pode, neste caso, ser de origem genética, e não ambiental. Os bovinos domésticos podem apresentar medo frente a novidades repentinas atenuadas com o passar dos anos de seleção. No entanto, o resultado encontrado pode ser interpretado como a não atenuação dessas respostas pelos animais do grupo NA, permanecendo ainda a resistência ao convívio com o homem (Price, 1984), principalmente quando estão impedidos de fugir por uma barreira física (Grandin \& Deesing, 1998).

Outros autores também encontraram diferenças de temperamento entre grupos genéticos. Grandin (2000) observou, em raças bovinas índicas, maior reatividade ao manejo. Voisinet et al. (1997a) registraram valores mais altos do escore comportamental de balança para os animais cruzados $(3,49 \pm 0,09 \times 1,80 \pm 0,10$ para os europeus $)(\mathrm{P}<0,001)$.

Os grupos genéticos diferiram quanto ao tempo de saída (TS) na primeira e quarta avaliações, nas quais os animais AA apresentaram maiores valores de TS que os NA (Tabela 2), talvez porque, durante a primeira avaliação, foram colocados

Tabela 1 - Valores médios de escore composto (EC) em cinco avaliações de bovinos Aberdeen Angus e Nelore $x$ Aberdeen Angus

Table 1 - Mean values of composite behavior score (BS) of Aberdeen Angus and Nellore $x$ Aberdeen Angus measured in five evaluations

\begin{tabular}{|c|c|c|c|}
\hline \multirow[t]{2}{*}{$\begin{array}{l}\text { Variável } \\
\text { Variable }\end{array}$} & \multicolumn{2}{|c|}{$\begin{array}{l}\text { Grupo genético } \\
\text { Genetic group }\end{array}$} & \multirow[t]{2}{*}{$\mathrm{P}>\mathrm{CMH}$} \\
\hline & Aberdeen Angus & $\begin{array}{c}\text { Nelore x Aberdeen } \\
\text { Angus }\end{array}$ & \\
\hline $\mathrm{EC} 1 \quad(B S 1)$ & $1,10 \pm 0,02$ & $3,20 \pm 0,02$ & 0,001 \\
\hline $\mathrm{EC} 2(B S 2)$ & $1,10 \pm 0,02$ & $2,60 \pm 0,02$ & 0,001 \\
\hline $\mathrm{EC} 3(B S 3)$ & $1,00 \pm 0,19$ & $1,95 \pm 0,19$ & 0,002 \\
\hline EC4 (BS4) & $1,10 \pm 0,13$ & $1,65 \pm 0,13$ & 0,006 \\
\hline $\mathrm{EC} 5(B S 5)$ & $1,00 \pm 0,14$ & $1,70 \pm 0,14$ & 0,002 \\
\hline EC6 (BS6) & 1 & 1 & 0,174 \\
\hline ECGlobal & - & - & 0,001 \\
\hline Overall $B S$ & & & \\
\hline
\end{tabular}

$\mathrm{CMH}$ - teste de Cochran-Mantel-Haenzel para verificar associação linear. $\mathrm{CMH}$ - Cochran-Mantel-Haenzel test to detect linear association. 
brincos de identificação e, na quarta, foram coletadas as amostras de sangue para a análise do cortisol, o que pode ter contribuído para exacerbar a reatividade dos animais, sem a modulação da experiência prévia. Em situações novas, o componente genético do temperamento fica mais aparente (Grandin, 1993), realçando as diferenças.

A variável ganho de peso se correlacionou negativamente com $\mathrm{ECm}(\mathrm{r}=-0,47, \mathrm{P}=0,0075, \mathrm{n}=30)$, mas positivamente $\operatorname{com} \operatorname{TSm}(r=0,39, \mathrm{P}=0,0336, \mathrm{n}=30)$, não ocorrendo diferença significativa quanto ao ganho de peso entre os grupos genéticos. Porém, foi observada diferença significativa dentro do grupo NA para a variável-resposta GMPT (ganho de peso médio total), na qual os animais menos reativos apresentaram maior ganho de peso, descrito pela seguinte equação: $\mathrm{GMDT}=0,55+0,11 \mathrm{TS} 1\left(\mathrm{P}=0,0410, \mathrm{R}^{2}=0,29\right)$. Esta diferença no grupo genético NA pode ser explicada pela maior amplitude dos valores de TS1 nos animais cruzados, indicando maior variabilidade desse grupo genético.

O maior ganho de peso dos animais mais calmos, em cada grupo genético (Voisinet et al, 1997a; Fell et al., 1999; Burns, 2003; Brown et al., 2004), foi relacionado por diversos autores ao maior consumo de matéria seca da dieta (Burns, 2003; Brown et al., 2004). Outros fatores foram considerados, mas não provados, como maior eficiência alimentar e menor incidência de doenças.

Os resultados deste estudo corroboram os descritos por Brown et al. (2004), que não constataram diferenças quanto à eficiência alimentar entre animais com valores extremos de velocidade de saída (VS). Essa característica

Tabela 2 - Valores médios do tempo de saída (TS), em segundos, e do ganho médio de peso, em $\mathrm{kg} / \mathrm{dia}$, de bovinos de dois grupos genéticos (Aberdeen Angus e Nelore $x$ Aberdeen Angus), medidos em cinco avaliações Table 2 - Mean values of the flight time test (FT, values in seconds) and of weight gain (WG, $\mathrm{kg} /$ day) of Aberdeen Angus and Nelore $x$ Aberdeen Angus measured in five evaluations

\begin{tabular}{lccc}
\hline Variável & \multicolumn{3}{c}{ Grupo genético } \\
Variable & \multicolumn{2}{c}{ Genetic group } & P $>\mathrm{F}$ \\
\cline { 2 - 3 } & Aberdeen Angus & Nelore x Aberdeen \\
Angus & \\
\hline TS1 (FT1) & $2,10 \pm 0,16$ & $1,34 \pm 0,16$ & 0,0022 \\
TS2 (FT2) & $2,61 \pm 0,32$ & $2,20 \pm 0,32$ & 0,3649 \\
TS3 (FT3) & $3,27 \pm 0,53$ & $2,60 \pm 0,53$ & 0,3739 \\
TS4 (FT4) & $4,67 \pm 0,57$ & $2,30 \pm 0,57$ & 0,0056 \\
TS5 (FT5) & $4,33 \pm 0,84$ & $3,16 \pm 0,84$ & 0,3261 \\
GMD1 (WG1) & $2,10 \pm 0,15$ & $1,59 \pm 0,15$ & 0,0203 \\
GMD2 (WG2) & $-0,57 \pm 0,17$ & $-0,40 \pm 0,17$ & 0,4860 \\
GMD3 (WG3) & $0,32 \pm 0,10$ & $0,29 \pm 0,10$ & 0,8600 \\
GMD4 (WG4) & $0,94 \pm 0,07$ & $1,05 \pm 0,07$ & 0,2714 \\
GMDT (TWG) & $0,78 \pm 0,04$ & $0,70 \pm 0,04$ & 0,1572 \\
\hline
\end{tabular}

poderia ser utilizada para compor um critério de seleção em programas de melhoramento genético Fordyce et al. (1988), o que, segundo Busby (2004), tem sido realizado por diversas associações de produtores nos Estados Unidos.

Esta proposta também foi feita por Burrow (1991), que calculou, pela média não-ponderada, herdabilidades de caracteres de temperamento, em distintos experimentos, de 0,36 e 0,23 para testes em que os animais não foram contidos e contidos, respectivamente, embora estas estimativas possam ser mais altas em animais mais jovens, sugerindo que a experiência prévia dos animais afeta seu temperamento e que a variação é reduzida aos animais adultos.

$\mathrm{Na}$ escolha de animais para programas de cruzamento, quando utilizado o teste de velocidade de fuga, percentuais de sangue zebuíno iguais ou superiores a $50 \%$ são indesejáveis se a característica temperamento é valorizada (Burrow $\&$ Corbet, 2000). Conforme Grandin (1993), em animais com temperamento extremo (muito agitados ou muito calmos), os escores tendem a se manter durante todo o tempo. No entanto, na avaliação de todos os animais, a medida deverá ser efetuada no primeiro contato com a nova situação para se determinar se essa medida expressa o genótipo ou a experiência prévia.

O escore composto e o tempo de saída foram altamente correlacionados (Tabela 3). Enquanto os diversos métodos de avaliação do temperamento podem descrever componentes diversos da reação de um animal ao manejo, sua alta correlação sugere mecanismos comuns a todos, como verificado também por Curley et al. (2004). Os coeficientes de correlações (Tabela 3) comprovam a estabilidade dos valores obtidos nas primeiras cinco avaliações.

Quanto à qualidade de carne, foram encontradas diferenças significativas de $\mathrm{pH}$ entre os grupos genéticos e entre as classes de temperamento. Os animais NA apresentaram valores de $\mathrm{pH} 1$ inferiores aos do grupo AA, embora essa diferença não tenha sido observada nos demais horários $(\mathrm{P}>0,05)$ (Tabela 4).

Os valores de $\mathrm{pH} 1$ médios de todos os animais podem ser considerados limítrofes, indicando que, independentemente do genótipo, todos os animais podem ter sofrido estresse agudo, com possível aumento da velocidade inicial do metabolismo post mortem. Ressalta-se que a temperatura das carcaças nesse horário foi de $39,3 \pm 1,64^{\circ} \mathrm{C}$, o que pode caracterizar desnaturação protéica.

Animais submetidos a situações de estresse agudo pouco antes ou durante o abate apresentam reações de defesa, que se manifestam por meio de contrações excessivas da musculatura, resultando em aumento do consumo de compostos energéticos do músculo, que provoca acelera- 
Tabela 3 - Coeficientes de correlação e seus respectivos valores de probabilidade entre o escore composto (EC) e o tempo de fuga (TS) medidos na primeira avaliação, em relação aos escores compostos medidos nas demais avaliações

Table 3 - Correlation coefficients and respective values of probability between composite behavior score (BS) and the flight time test (FT) measured in the first evaluation compared to the composed scores measured in other evaluations

\begin{tabular}{|c|c|c|}
\hline $\begin{array}{l}\text { Variável } \\
\text { Variable }\end{array}$ & $\begin{array}{c}\text { Coeficiente de correlação } \\
\text { Correlation coefficient }\end{array}$ & $\mathrm{P}>\mathrm{t}$ \\
\hline $\mathrm{EC} 1 \times \mathrm{EC} 2(B S 1 \times B S 2)$ & 0,69 & 0,0001 \\
\hline $\mathrm{EC} 1 \times \mathrm{EC} 3\left(\begin{array}{lll}B S 1 & x S 3)\end{array}\right.$ & 0,58 & 0,0001 \\
\hline $\mathrm{EC} 1 \times \mathrm{EC} 4\left(\begin{array}{llll}B S 1 & x & B S 4\end{array}\right)$ & 0,60 & 0,0001 \\
\hline $\mathrm{EC} 1 \times \operatorname{EC} 5(B S 1 \times B S 5)$ & 0,64 & 0,0001 \\
\hline $\mathrm{EC} 1 \times \mathrm{EC} 6(B S 1 \times B S 6)$ & 0,34 & 0,0690 \\
\hline $\mathrm{EC} 1 \times \mathrm{ECM}(B S 1 \times M B S)$ & 0,86 & 0,0001 \\
\hline $\mathrm{TS} 1 \times \mathrm{EC} 1\left(\begin{array}{lll}F T 1 & x & B S 1)\end{array}\right.$ & $-0,55$ & 0,0003 \\
\hline $\mathrm{TS} 1 \times \mathrm{EC} 2\left(\begin{array}{lll}F T 1 & x B S 2\end{array}\right)$ & $-0,55$ & 0,0002 \\
\hline $\mathrm{TS} 1 \times \mathrm{EC} 3\left(\begin{array}{lll}F T 1 & x \mathrm{BS} 3\end{array}\right)$ & $-0,38$ & 0,0341 \\
\hline TS1 x EC4 ( $\left.\begin{array}{llll}F T 1 & x & B S 4\end{array}\right)$ & $-0,39$ & 0,0123 \\
\hline $\mathrm{TS} 1 \times \operatorname{EC} 5(F T 1 \times B S 5)$ & $-0,35$ & 0,0263 \\
\hline TS1 x EC6 (FT1 $x$ BS6) & $-0,29$ & 0,1100 \\
\hline $\mathrm{TS} 1 \times \mathrm{ECM}(F T 1 \times M B S)$ & $-0,60$ & 0,0005 \\
\hline TSM x EC1 (MFT $x$ BSI) & $-0,54$ & 0,0019 \\
\hline TSM $\times$ EC2 (MFT $x$ BS2) & $-0,59$ & 0,0019 \\
\hline TSM x EC3 (MFT $x$ BS3) & $-0,36$ & 0,0513 \\
\hline
\end{tabular}

Tabela 4 - Valores médios de $\mathrm{pH}$ post mortem de carcaças bovinas, medidos 1, 3, 7, 12 e 24 horas após o abate, de acordo com o grupo genético

Table 4 - Mean values of post mortem $\mathrm{pH}$ measured in bovine carcasses at 1, 3, 7, 12 and 24 hours after slaughter according to the genetic group

\begin{tabular}{|c|c|c|c|c|c|}
\hline $\begin{array}{l}\text { Grupo genético } \\
\text { Genetic group }\end{array}$ & pH 1 & pH3 & $\mathrm{pH} 7$ & pH 12 & pH24 \\
\hline Aberdeen Angus & 6,58 a & 6,14 a & 5,90 a & 5,69 a & $5,54 \mathrm{a}$ \\
\hline $\begin{array}{l}\text { Nelore x Aberdeen } \\
\text { Angus }\end{array}$ & $6,17 \mathrm{~b}$ & $6,01 \mathrm{a}$ & 5,83 a & 5,65 a & 5,53 a \\
\hline
\end{tabular}

a,b médias na mesma coluna seguidas de letras distintas diferem significativamente segundo o teste $F(P<0,05)$.

$a, b$ means in the same column followed by distinct letters differ $(P<0.05)$ according to F-test.

ção da glicólise anaeróbia (Champe \& Harvey, 1996; Lehninger, 1986). Conseqüentemente, a velocidade de produção do lactato aumenta (o pH passa para de 7,0 5,4-5,5) e as mudanças que ocorreriam normalmente em 12 horas, ocorrem em 90, 30 e até 10 minutos. Por outro lado, o estresse crônico prejudica a circulação sangüínea, ocasionando deficiência de oxigênio no músculo e acarretando intensa hidrólise do ATP. A velocidade de redução do pH e o valor do $\mathrm{pH}$ final da carne após 24 horas, em bovinos, normalmente desenvolvem-se lentamente: o $\mathrm{pH}$ inicial (0 hora) diminui de 7,0 para 6,4-6,8 (após 5 horas) e para 5,5-5,9 (após 24 horas).

Considerando o efeito do grupo genético, os valores de pH1 no grupo AA indicam velocidade de glicólise anaeróbia intermediária, ou seja, nos padrões considerados ainda normais para o metabolismo post mortem, enquanto, no grupo NA, essa velocidade foi mais rápida.

Barbosa et al. (2000) também verificaram valores de $\mathrm{pH} 1$ inferiores para bovinos cruzas zebuínos em relação aos europeus. Os valores de $\mathrm{pH}$ encontrados neste trabalho são similares aos verificados por Hwang \& Thompson (2001) em carcaças previamente estimuladas após a sangria. Zamora et al. (1996), em estudo com bovinos da raça Charolês, registraram valores de $\mathrm{pH}$ uma hora post mortem similares aos verificados no grupo genético AA.

Os animais mais reativos apresentaram valores de $\mathrm{pH} 1$ significativamente inferiores aos dos animais calmos, mas essa diferença não foi mantida nos demais horários post mortem avaliados (Tabelas 5 e 6 ).

Esses resultados podem ser decorrentes do estresse agudo causado pelo manejo pré-abate, uma vez que os procedimentos se constituem per se em fatores estressantes muito fortes para os animais (Grandin, 1994).

Petherick et al. (2002), constataram que animais submetidos ao sistema de manejo menos afável e com pior temperamento apresentaram evidências de $\mathrm{pH}$ inicial menor. Voisinet et al. (1997b), no entanto, verificaram, em bovinos de raças sintéticas, que animais com temperamento mais excitável possuem carne mais escura ao corte, segundo USDA, após o abate, reduzindo sua qualidade. Animais com temperamento nervoso podem tornar-se excitados e excessivamente estressados, o que, no momento do abate, pode alterar o $\mathrm{pH}$ da carcaça, afetando as características qualitativas e organolépticas da carne (Fordyce et al., 1988).

Não foram constatadas diferenças significativas na concentração de cortisol entre os grupos genéticos estuda-

Tabela 5 - Valores médios de $\mathrm{pH}$ post mortem de carcaças bovinas medidos $1,3,7,12$ e 24 horas após o abate, de acordo com o escore comportamental (EC)

Table 5 - Mean values of post mortem $\mathrm{pH}$ measured in bovine carcasses at 1, 3, 7, 12 and 24 hours after slaughter according to the composite behavior score (BS)

\begin{tabular}{llllll}
\hline $\mathrm{EC}$ & $\mathrm{pH} 1$ & $\mathrm{pH} 3$ & $\mathrm{pH} 7$ & $\mathrm{pH} 12$ & $\mathrm{pH} 24$ \\
$B S$ & & & & & \\
\hline 1 (Manso) & $6,47 \mathrm{a}$ & $6,11 \mathrm{a}$ & $5,91 \mathrm{a}$ & $5,69 \mathrm{ab}$ & $5,53 \mathrm{a}$ \\
1 (Docile) & & & & & \\
2 (Intermediário) & $6,28 \mathrm{~b}$ & $6,02 \mathrm{a}$ & $5,86 \mathrm{a}$ & $5,48 \mathrm{~b}$ & $5,41 \mathrm{a}$ \\
2 (Intermediary) & & & & & \\
3 (Nervoso) & $6,09 \mathrm{c}$ & $6,01 \mathrm{a}$ & $5,83 \mathrm{a}$ & $5,75 \mathrm{a}$ & $5,62 \mathrm{a}$ \\
3 (Nervous) & & & & &
\end{tabular}

a,b Médias na mesma coluna seguidas de letras distintas diferem significativamente $(P<0,05)$ segundo o teste DMS Fisher.

$a, b$ Means in the same column followed by distinct letters differ $(P<0.05)$ according to FisherDMS test. 
Tabela 6 - Valores médios de pH post mortem de carcaças bovinas medidos 1, 3, 7, 12 e 24 horas após o abate, de acordo com o tempo de saída (TS)

Table 6 - $\quad$ Mean values of post mortem $\mathrm{pH}$ measured in bovine carcasses at 1, 3, 7, 12 and 24 hours after slaughter according to the flight time test (FT)

\begin{tabular}{|c|c|c|c|c|c|}
\hline $\begin{array}{l}\mathrm{TS} \\
F T\end{array}$ & pH 1 & pH3 & p H 7 & pH 12 & $\mathrm{pH} 24$ \\
\hline $\begin{array}{l}1 \text { (Mais rápida) } \\
1 \text { (Fast) }\end{array}$ & $6,10 \mathrm{~b}$ & $6,04 a$ & $5,87 \mathrm{a}$ & $5,73 \mathrm{a}$ & $5,61 \mathrm{a}$ \\
\hline $\begin{array}{l}2 \text { (Intermediária) } \\
2 \text { (Intermediary) }\end{array}$ & $6,43 a$ & $6,06 \mathrm{a}$ & $5,83 \mathrm{a}$ & $5,58 \mathrm{a}$ & $5,43 a$ \\
\hline $\begin{array}{l}3 \text { (Mais lenta) } \\
3 \text { (Slow) }\end{array}$ & $6,43 \mathrm{a}$ & $6,09 a$ & $5,98 \mathrm{a}$ & $5,74 a$ & $5,62 \mathrm{a}$ \\
\hline
\end{tabular}

dos, com valores médios de $52+13,18 \mathrm{ng} / \mathrm{mL}$, mínimos de $29 \mathrm{ng} / \mathrm{mL}$ e máximos de $71 \mathrm{ng} / \mathrm{mL}$ (Tabela 7).

Segundo Grandin (1997), os valores encontrados estão acima daqueles considerados normais para bovinos $(15 \mathrm{ng} / \mathrm{mL})$ durante o processo de abate, considerando-se o animal contido com calma e insensibilizado imediatamente após um só disparo da pistola de dardo retráctil. Zavy et al. (1992) encontraram, em bezerros cruza Bos taurus indicus, valores basais de cortisol plasmático $26 \%$ maiores que em Bos taurus taurus após o transporte. Curley et al. (2004), ao estudarem novilhas Brahman, classificaram-nas como reativas $(48,97$ $\pm 3,42 \mathrm{ng} / \mathrm{mL})$ ou calmas $(29,60 \pm 5,46 \mathrm{ng} / \mathrm{mL}$ de cortisol).

Não foram observadas diferenças significativas de cor $\mathrm{L}^{*}$ $a^{*}, b^{*}$ e o ângulo $H^{\circ}$ entre os dois grupos genéticos estudados, havendo apenas tendência de animais pertencentes ao grupo genético NA apresentar cor mais escura (Tabela 8).

Houve interação significativa grupo genético $\times$ TS para cor a* e b*. Não foram detectadas, no entanto, diferenças de cor $a^{*} \mathrm{e} b *$ na carne entre os animais AA que apresentaram valores de velocidade de saída distintos. Porém, a carne nos novilhos NA apresentou valores $23 \%$ maiores de cor a* $(\mathrm{P}=0,0394)$ e $9 \%$ maiores de cor $\mathrm{b}^{*}(\mathrm{P}=0,0808)$ nos animais com maior valor de TS. A cor $\mathrm{L}^{*}$ esteve negativamente correlacionada a EC e pH $24(\mathrm{r}=-0,54$ e $-0,39$, respectivamente) e a cor $\mathrm{b}^{*}$, com pH3 e pH7 ( $\mathrm{r}=-0,39$ e $-0,45$, respectivamente). Os valores dos parâmetros $\mathrm{L}^{*}, \mathrm{a}^{*}, \mathrm{~b}^{*}$ dos animais NA foram menores, o que pode indicar cor vermelha mais escura. Esta tendência para a cor vermelha mais escura também foi observada por Wulf et al. (1997), ao avaliarem novilhos Bos taurus taurus e Bos taurus indicus.

$\mathrm{O} \mathrm{pH} 1$ diminuiu com o aumento do $\mathrm{EC}$ e do ângulo $\mathrm{H}^{\circ}$, segundo a seguinte equação de regressão: $\mathrm{pH} 1=7,30$ $0,284 \mathrm{ec}-0,11 \mathrm{H}^{\circ} \mathrm{ab}\left(\mathrm{P}=0,0001, \mathrm{R}^{2}=0,92\right)$. Maiores valores de EC ou menores de TS indicam maior reatividade e movi-
Tabela 7 - Valores médios de cortisol plasmático de bovinos de dois grupos genéticos (Aberdeen Angus - AA e Nelore x Aberdeen Angus - NA)

Table 7 - Mean values of plasmatic cortisol according to two genetic groups Aberdeen Angus (AA) and Nelore x Aberdeen Angus (NA)

\begin{tabular}{lccc}
\hline & \multicolumn{2}{c}{$\begin{array}{c}\text { Grupo genético } \\
\text { Genetic group }\end{array}$} & \\
\cline { 2 - 3 } & AA & NA & P > F \\
\hline $\begin{array}{l}\text { Nível de cortisol }(\mathrm{ng} / \mathrm{mL}) \\
\text { Cortisol level }(\mathrm{ng} / \mathrm{mL})\end{array}$ & 52,25 & 51,83 & 0,9643 \\
\hline
\end{tabular}

Tabela 8 - Valores médios de cor, segundo o sistema CIELab, da carne do músculo Longissimus dorsi de novilhos Aberdeen angus (AA) e Nelore $x$ Aberdeen Angus (NA)

Table 8 - $\quad$ Mean values of meat color measured in the longissimus dorsi of Aberdeen angus (AA) and Nelore $x$ Aberdeen Angus (NA) steers according to the CIELab system

\begin{tabular}{lccc}
\hline & \multicolumn{2}{c}{$\begin{array}{c}\text { Grupo genético } \\
\text { Genetic }\end{array}$} & \\
\cline { 2 - 3 } & AAroup & \\
\hline Cor L* $^{*}$ Color $\left.L^{*}\right)$ & 39,157 & NA & P $>$ F \\
Cor a* $\left(\right.$ Color $\left.a^{*}\right)$ & 20,220 & 19,770 & 0,6461 \\
Cor b* $\left(\right.$ Color $\left.b^{*}\right)$ & 4,6390 & 4,4695 & 0,1582 \\
${\text { Ângulo } \mathrm{H}^{\circ}\left(\text { Angle } H^{\circ}\right)}^{12,798}$ & 12,158 & 0,7601 \\
\hline
\end{tabular}

mentação animal, o que pode resultar em aumento da taxa de consumo de glicogênio, diminuindo o pH inicial.

\section{Conclusões}

O genótipo e o temperamento em bovinos de corte podem afetar características de interesse econômico, como o ganho de peso dos animais e a qualidade final da carne, pois bovinos Nelore x Angus mais reativos apresentaram menor ganho de peso e maior velocidade inicial da glicólise anaeróbia.

\section{Literatura Citada}

ABULARACH, M.L.S.; ROCHA, C.E.; FELÍCIO, P.E. Características de qualidade do contrafilé (músculo longissimus dorsi) de touros jovens da raça Nelore. Ciência e Tecnologia de Alimentos, v.18, n.2, p.205-210, 1998.

AGUILAR, N.M.A.; BALBUENA, O.; PARANHOS DA COSTA, M.J.R. Evaluacion del temperamento em bovinos cruza cebú. In: ENCONTRO ANUAL DE ETOLOGIA - Comportamento e Desenvolvimento Sustentável, 22., 2004, Campo Grande. Anais... Campo Grande: Sociedade Brasileira de Etologia/Elohim reproduções, 2004, CD-ROM.

ANUALPEC - Anuário Estatístico da Pecuária de Corte. São Paulo. FNP (Consultoria/Agroinformativos), p.77-101, 2003 . 
BARBOSA, I.D.; OSÓRIO, M.T.M.; SOARES, GJ.D Influência da genética de bovinos na velocidade do metabolismo post mortem. Revista Brasileira de Agrociência, v.6, n.3, p.243-247, 2000.

BENDALL, J.R. Post mortem changes in muscle. In: BOURNE, G.H. (Ed.) The structure and functions of muscle. 2.ed. New York: Academic Press, 1973. v.2, p.244.

BOISSY, A.; BOUISSOU, M. F. Assessment of individual differences in behavioral reactions of heifers exposed to various feareliciting situations. Applied Animal Behaviour Science, v.46, p.17-31, 1995.

BORBA, L.H.F.; PIOVESAN, U.; PARANHOS DA COSTA, M.J.R. Uma abordagem preliminar no estudo de associação entre escores de reatividade e características produtivas de bovinos de corte. Anais de Etologia, v.15, p.388, 1997.

BROWN, E.G.; CARSTENS, G.E.; FOX, J.T. et al. 2004. Relationships between temperament and performance traits of growing calves. In: Beef Cattle Research in Texas Publication, section Physiology. Disponível em: www.animalscience.tamv.edu/ansc/beef/bcrt/2004/brown $>$. Acesso em: $12 / 12 / 2004$

BURNS, R. Study shows temperamental cattle eat less, gain less. AgNews Agricultural Communications, v.30, p.45-49, 2003.

BURROW, H.M.; SEIFERT, G.W.; COBERT, N.J. A new technique for measuaring temperament in cattle. Australian Society of Animal Production, v.17, p.154-157, 1988.

BURROW, H.M. Effect of intensive handling of zebu crossbred weaner calves on temperament. In: CONFERENCE OF THE AUSTRALIAN ASSOCIATION OF ANIMAL BREEDING AND GENETICS, 1991, Victoria. Proceedings... Victoria, 1991. v.9, p.208-211.

BURROW, H. M.; CORBET, N. J. Genetic and environmental factors affecting temperament of zebu and zebu-derived beef cattle grazed at pasture in the tropics. Australian Journal of Agricultural Research, v.51, p.155-162, 2000.

BURROW, H.M.; DILLON, R.D. Relationship between temperament and growth in a feedlot and commercial carcass traits of Bos indicus crossbreeds. Australian Journal Experimental Agriculture, v.37, p.407-411, 1997.

BUSBY, D. Disposition - convenience trait or economically important. 2004. Disponível em: <www. xtension.iastate.Edu/ feci/4stbeef/disposition.pdf $>$.Acesso em: 15/12/2004.

CHAMPE, P.C.; HARVEY, R.A. Bioquímica ilustrada. 2.ed. Porto Alegre: Artes Médicas, 1996. 446p.

CURLEY JR., K.O.; NEUENDORFF, D.A.; LEWIS, A.W et al. Evaluation of temperament and stress physiology may be useful in breeding programs. In: Beef Cattle Research in Texas Publication, section Physiology. Disponível em: www.animalscience.tamv.edu/ansc/beef/bcrt/2004/curley> Acesso em: 05/01/2005.

FELL, L.R.; COLDITZ, I.G.; WALKER, K.H. et al. Associations between temperament, performance and immune function in cattle entering a commercial feedlot. Australian Journal of Experimental Agriculture, v.39, p.795-802, 1999.

FORDYCE, G.; DODT, R.M.; WHYTES, J.R. Cattle temperaments in extensive beef herds in northern Queensland - Factors affecting temperament. Australian Journal of Experimental Agriculture, v.28, p.683-687, 1988.

GRANDIN, T. Assessment of stress during handling and transport. Journal of Animal Science, v.75, 249-257, 1997.

GRANDIN, T. Behavioral agitation during handling of cattle is persistent over time. Applied Animal Behaviour Science, v.36, p.1-9, 1993.

GRANDIN, T. Livestock handling and transport. Wallingford: CABI Publishing, 2000. p.63-85.

GRANDIN, T. Methods to reduce PSE and bloodplash. Allen D. Leman Swine Conference, v.21, p.206-209, 1994.

GRANDIN, T.; DEESING, D. Genetics and behavioural of domestic animals. San Diego: Academic Press, 1998. 378p.
HOUBEN, J.H.; DIJK, A.; EIKELENBOOM, G. et al. Effect of dietary vitamin E supplementation, fat level and packaging on colour stability and lipid oxidation in minced beef. Meat Science, v.55, p.331-336, 2000.

HWANG, I.H.; THOMPSON, J.M. The effect of time and type of electrical stimulation on the calpain system and meat tenderness in beef Longissimus dorsi muscle. Meat Science, v.58, p.135-144, 2001.

KILGOUR, R. The open field-test as an assessment of the temperament of dairy cows. Animal Behaviour, v.23, p.615-624, 1998.

LAWRIE, R.A. Ciencia de la carne. 3.ed. Zaragoza: Acribia, 1998. 367p.

LEHNinger, A.L. Princípios da bioquímica. 3.ed. São Paulo: Sarvier, 1986. $725 \mathrm{p}$.

MOBERG, G.P. Biological response to stress: implications for animal welfare. In: MOBERG, G.P.; MENCH, J.A. (Eds.). The biology of animal stress: basic principles and implications for animal welfare. Wallingford: CABI Publishing, 2000. p.1-22,

PARANHOS DA COSTA, M.J.R.; PIOVESAN, U.; CYRILLO, J.N. S.G. et al. In: WORLD CONGRESS ON GENETICS APPLIED TO LIVESTOCK PRODUCTION, 7., Montpellier, 2002. Proceedings... Montpellier: INRA, 2002. v.7, p.5.

PETHERICK, J.C.; HOLROYD, R.G.; DOOGAN, V.J. et al. Productivity, carcass and meat quality of lot fed Bos indicus cross steers grouped according to temperament. Australian Journal of Experimental Agriculture, v.42, n.4, p.389-398, 2002.

PIOVESAN, U. Análise de fatores genéticos e ambientais na reatividade de quatro raças de bovinos de corte ao manejo. Jaboticabal: Universidade Estadual Paulista, 1998. 42p. Dissertação (Mestrado em Zootecnia) - Universidade Estadual Paulista, 1998

PRICE, E.O. Behavioral aspects of animal domestication. Quimical Review Biological, v.59, p.1-32, 1984.

ROGAN, M.T.; LeDOUX, J.E. Emotion: Systems, cells and synaptic plasticity. Cell Press, v.85, p.469-475, 1996.

STATISTICAL ANALYSES SYSTEM - SAS. SAS/STAT ${ }^{\circledR}$ User's Guide, Version 6, 4.ed., Cary: 1989. v.2, 846p.

VOISINET, B.D.; GRANDIN, T.; TATUM, J.D. et al. Feedlot cattle with calm temperaments have higher average daily gains than cattle with excitable temperaments. Journal of Animal Science, v.75, p.892-896, 1997a.

VOISINET, B.D.; GRANDIN, T.; O'CONNOR, S.F. et al. Bos indicus-cross feedlot cattle with excitable temperaments have tougher meat and a higher incidence of borderline dark cutters. Meat Science, v.46, p.367-377, 1997b.

WULF, D.M.; O'CONNOR, S.F.; TATUM, J.D. et al. Using objective measures of muscle color to predict beef longissimus tenderness. Journal of Animal Science, v.75, p.684-692, 1997.

ZAMORA, F; DEBITONS, E.; LEPETIT, J. et al. Predictions variability of ageing and toughness in beef M. Longissimus lumborum et thoracis. Meat Science, v.43, n.3-4, p.321-333, 1996.

ZAVY, M.T.; JUNIEWICZ, P.E.; PHILLIPS, W.A et al. Effect of initial restraint, weaning, and transport stress on baseline and ACTH-stimulated cortisol responses in beef calves of different genotypes. Animal Journal Veterinary Research, v.53, n.4, p.551-557, 1992. 